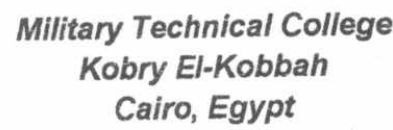

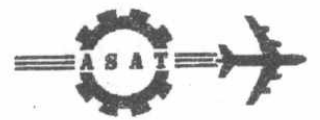

$10^{\text {th }}$ International Conference

On Aerospace Sciences\&

Aviation Technology

\title{
Classical Autopilot Design for a Semiactive Homing Guided Missile
}

\author{
A.M. Kamel, G. A. El-Sheikh and I. Abd-EIDayem
}

\begin{abstract}
The control system of a guided missile is composed of the missile itself, equipped with an automatic pilot, and a guidance system, which is either on the missile or outside of it. The guidance system measures the missiles coordinates and out of them in addition to the target coordinates creates the control signals for deflecting the fins in such a direction that should result in the minimum deflection of the missile from the required trajectory. The main requirement imposed on the missile autopilot is the transfer of the control signals into mechanical rotation of the control fins. During missile flight, it is necessary to keep the missile's dynamic properties approximately fixed or slowly varying. Thus, any undue changes in the missile's maneuverability can be avoided using feedbacks from the angular speed and from the missile's normal acceleration to stabilize and to improve the dynamic properties of the missile and of the whole control circuit. Therefore, this paper is devoted to investigate the performance of a homing guided missile using the available compensators about that system. Then, a trial is followed to design another compensator or correction networks for enhancing the missile system performance. The performance of the guided missile is measured through the minimum miss distance and the speed of response.
\end{abstract}

Keywords: Missile Guidance and Classical Control

\section{1- Introduction}

The underlying missile is a medium range anti-aircraft missile, with a boost phase followed by a sustain phase, and is aerodynamically controlled via two pairs of control wings. It is a single stage missile, but once the solid boost propellant is consumed, the resulting empty chamber is used for a ramjet propulsion system. At the end of boost, the tail section cap is discarded, and the air intake covers are sucked in and expelled out the rear of the missile. The flight sequence is boost followed by sustain followed by a power off portion.

The problem of analyzing and designing guidance law and flight control systems (autopilot) for guided missiles is simply a control problem. The guidance and control sysiem supplies steering commands to control surfaces so as to point the missile 
towards its target with minimum maneuver. The main loop within the guidance and control process is the autopilot, which is a closed loop consisting of inertial sensors such as accelerometers and/or gyros in addition to control or compensation networks. According to the plane of control action, the autopilot may be called lateral or roll autopilot. One of the main objectives for autopilots is to maintain near-constant steady state aerodynamic gain. In addition, it can be used to reduce the cross coupling between pitch and yaw motions. This cross coupling effect can be regarded as disturbances and the closed loop system should be considerably less sensitive to them.

Three of the principal requirements of a good autopilot are quick response with minimum acceleration error, stability, and robustness. When the autopilot calls for a control deflection to achieve a lateral maneuver, it takes time to move the control surface into the required position against the possible resisting aerodynamic torque and the inertia of that surface. It also takes time for the control moment to move the missile into the required angle of attack for the required maneuver. These factors affect the capability of the autopilot to achieve a quick response.

Generally, it is desired to have a fast response with minimum overshoot so that the maneuver is attained quickly and is of the desired magnitude. Minimum overshoot is practically important at high acceleration levels to avoid exceeding structural limitations, and the minimum acceleration error is necessary for effective guidance. Another important requirement for the autopilot is the stability, i.e. the capability of the missile to return to an equilibrium condition after sustaining a disturbance. This requirement does not impose a requirement of positive aerodynamic stability but rather requires that the autopilot contain sufficient feedback loops to provide an equivalent aerodynamic stability. In addition, the autopilot should provide reasonable attenuation of high frequencies so that it does not respond to high-frequency aero-elastic behavior that can affect the sensor signals nor to noise content in the acceleration commands.

The designed autopilot should handle adequately broad variations of the aerodynamic parameters of the missile configuration i.e. it is robust. This necessitates the aerodynamic description of the missile configuration over the full operating range of altitude. Mach number, angles of attack and sideslip, and control deflections in pitch, yaw, and roll. The accelerometers and gyroscopes that measure the missile dynamics must be placed in positions that permit them to register the complete dynamics of the missile without being too greatly affected by local dynamic behavior. The accelerometers should be placed in positions where the translational vibrations of the body are minimal, while the pitch and yaw rate sensors should be placed where the rotational vibrations are minimal [2].

\section{2- Guidance System Simulation}

Simulation is a process of imitating the behavior of the actual missile system in a stepwise and concise approach. The set of physical equations governing the guided missile motion are solved numerically on computers yielding a flexible and reliable tool for system design and analysis. Once the guidance system is designed, the behavior of different equipments is proved through flight tests from which the data are collected utilizing telemetry systems. These data are then evaluated to furnish an additional aid to the designer of the guidance system. This evaluation process and redesign are carried out utilizing the simulation computers, which are considered main components 
within the missile guidance system during the design and implementation phases. Thus, toward the objective of this paper a computer code is written within MATLAB environment to simulate the different missile-target engagement scenarios.

\subsection{Reference Frames and Coordinate Transformations}

Due to the nature of the underlying problem and to have a datum for measuring the flight variables, different reference frames should be mechanized. Among these reference frames are the body, velocity, and the ground coordinate systems, as shown in Fig. (1). The simulation incorporates a simplified earth and gravity model, where a flat, non-rotating earth is assumed as the flight duration is typically less than one minute. Similarly, a constant magnitude gravitational potential acting downward along the local vertical vector is implemented.

The mutual position between different coordinate systems can be expressed using one of three tenuous: Direction cosine, Euler's angles, and Quaternion parameters. The quaternion approach is preferred as it expresses the entries in the array as algebraic quantities rather than as trigonometric functions, which are more time consuming to calculate, and have numerical singularities. Once the matrix is obtained, the different flight variables are transformed from the parent coordinate system to the specified inertial reference coordinate system, yielding the correct quantities that can be used together in guidance calculations. The launch parameters are used to define the orientation of the missile coordinate systems relative to the earth reference system in the form of initial quaternions.
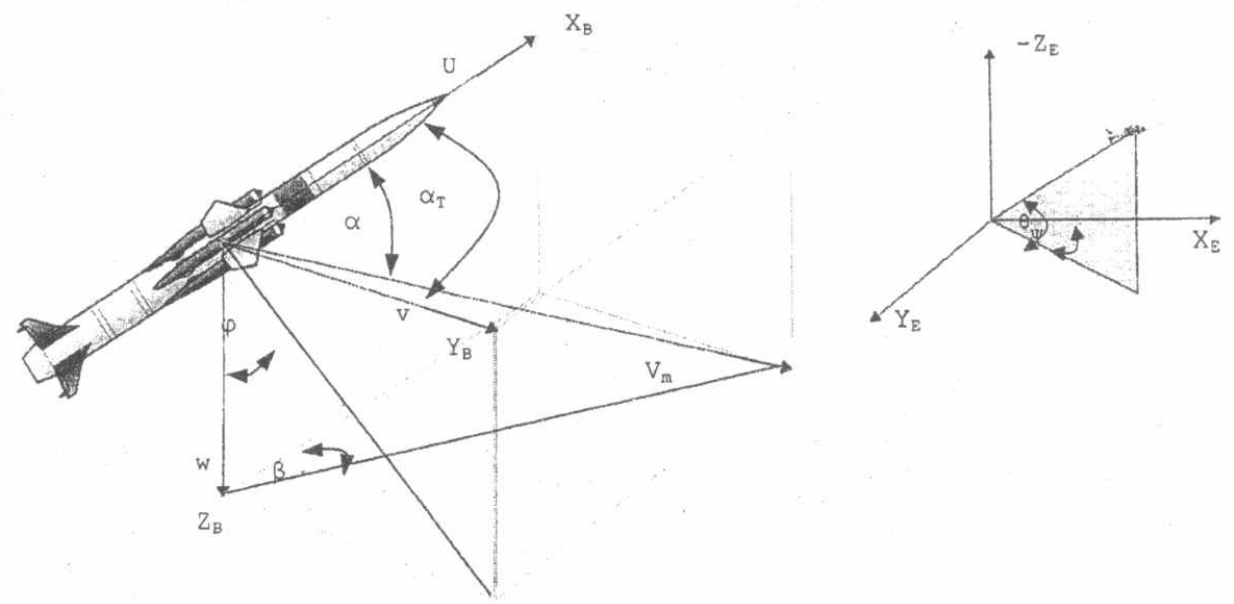

Fig. (1): Missile BCS and velocity components

\subsection{Dynamics of Missile Motion}

The missile flies in space under the effect of thrust, weight, and aerodynamic forces. The action of these forces has a certain effect on the shape of the missile trajectory. The change of missile velocity $\left(\overline{\mathrm{V}}_{\mathrm{m}}\right)$ direction is performed with the help of aerodynamic forces in the atmosphere. To quantify these changes and clarify the missile performance during its flight, the equations of motion are derived by applying Newton's laws of motion, which relate the summation of the external forces and 
moments to the linear and angular missile accelerations. Toward this objective, an inertial reference frame is defined and utilized during the computation/guidance process [13].

The engine thrust is determined by means of reaction forces due to the action of the exhaust upon the atmosphere. It is usually distributed into axes of the body coordinate system with the main basic component lies along the longitudinal axis of the missile [12]. For the underlying system, the first flight stage is powered by a solid propellant booster, and the second portion of

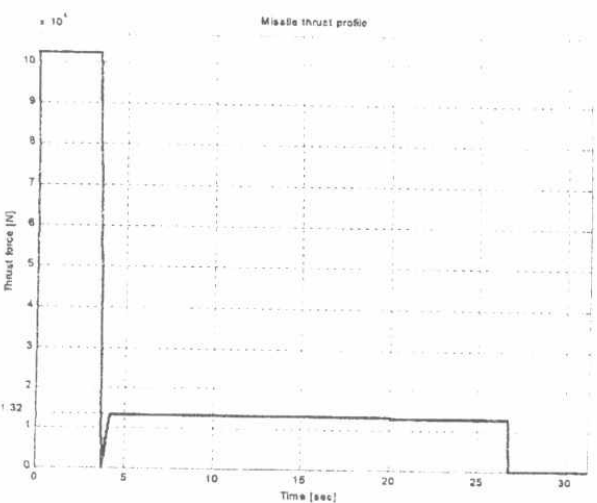

Fig. (2): Missile Thrust Force Profile the flight is powered by a ramjet engine. The thrust profile was portrayed with ramps and constant functions as shown in Fig. (2).

The Aerodynamic force is usually distributed into axes of the velocity coordinate system, which are related to the direction of the missile motion. The components of this force are resolved along the missile body axes as $F_{x a}, F_{y a}$, and $F_{z a}$. These force components create aerodynamic moments owing to the fact that they do not pass through the missile center of gravity. The aerodynamic moment components around the missile body axes are $M_{x a}, M_{y a}$, and $M_{z a}$. In case of thrust misalignment, a thrust moment will be created and should be taken into consideration.

Toward the objective of the paper, a 6DOF model is built and simulation is conducted using the above data. One of the engagement scenarios is considered as the nominal case, in

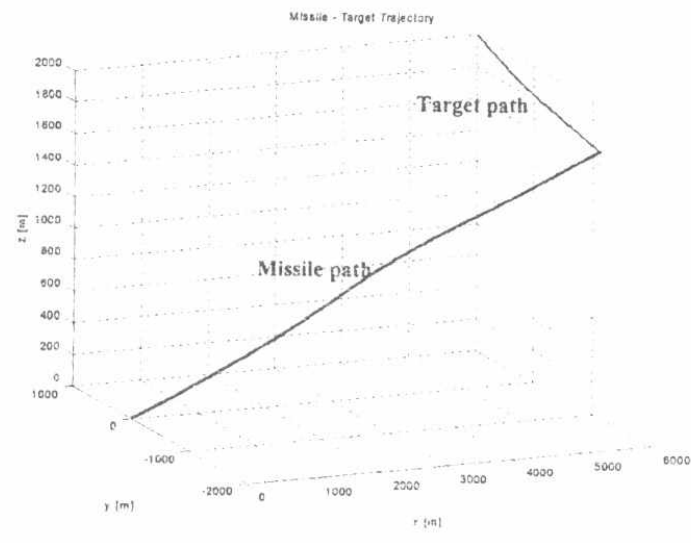

Fig. (3): Missile - Target engagement scenario which the target maneuver was about $3 g$ away from the missile. In addition, the maneuver start-time is early so that the missile could follow the target easily. The obtained flight paths for the target and missile are shown in Fig. (3).

\section{3- Missile Control System}

The missile control system is used to control the missile by executing the guidance commands and to correct its trajectory through control surfaces deflections. The underlying system consists of three channels; pitch channel, yaw channel, and roll channel. Each channel consists of linear accelerometer, damping gyro, shaping circuits, and the control fin drive system. The electrical signals are amplified and 
conditioned within the control loop of each channel, where the control surfaces deviate in the direction that interlocks the input signal. The control system performance of the underlying missile has been investigated through its structure and aerodynamics transfer function.

\subsection{Control System Structure}

Many different guidance laws have been exploited based on various design concepts over the years. The most popular guidance laws involve LOS rate guidance, which is referred as proportional navigation guidance (PNG). That is, guidance commands proportional to the LOS angle rate are used to correct the missile course via the guidance loop. Generally, the flight control system used in homing missiles is a threeloop autopilot, including a rate loop, an accelerometer, and a synthetic stability loop [6]. The underlying system autopilot consists of three channels used to control the missile attitude and attitude rate in pitch, yaw, and roll planes in addition to acceleration control system in pitch and yaw planes. Both pitch and yaw channels in the control system have the same structure, as shown in Fig. (4). It is clear that the transfer dynamics of the missile airframe should be available for autopilot analysis and design.

The proportional navigation guidance law is considered in which, the rate of change of missile velocity direction is proportional to the rate of change of the missile-target line of sight (LOS) as follows [2]:

$\dot{\gamma}=\mathrm{N} \dot{\sigma}$

where: $y$ is the flight path angle, and $\sigma$ is the line of sight angle, and $N$ is a design constant greater than unity and is defined as the navigation gain. The seeker antenna detects the angle between the LOS and the antenna axis (radar antenna error angle $(\varepsilon)$ ), and the radar receiver produces an output voltage $\left(V_{R}\right)$, proportional to this angle which has the form $V_{R}=K_{R} \varepsilon$, with $K_{R}$ representing the gain of seeker receiver. Due to the geometry shown in Fig. (5), the antenna position, the line of sight, and the antenna axis are related by the following equation:

$\varepsilon=\sigma-(\theta+\lambda)$

where: $\theta$ is the angular position of longitudinal axis, and $\lambda$ is the angular orientation of radar antenna axis w.r.t. the missile longitudinal axis.

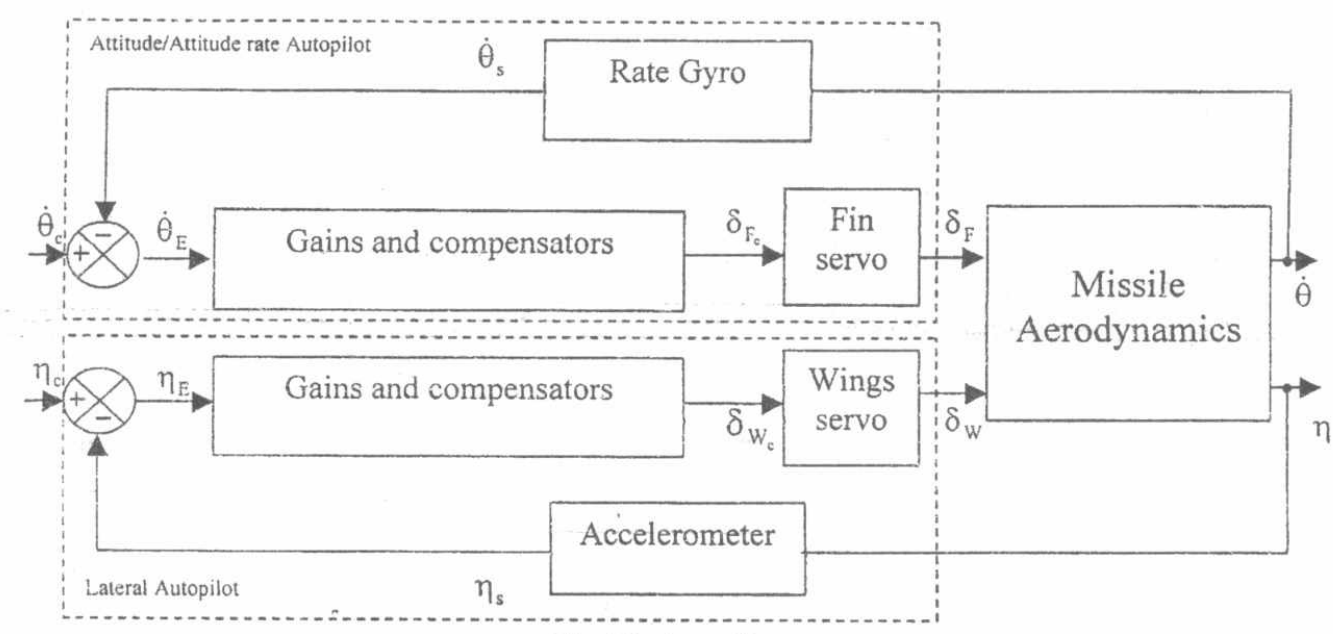

Fig. (4): Autopilot structure 
Ideally, the antenna can be rotated such that its angular velocity $(\dot{\theta}+\dot{\lambda})$ is proportional to the radar receiver output voltage $V_{R}$ i.e.

$\dot{\theta}+\dot{\lambda}=\mathrm{KV}_{\mathrm{R}}$

where $K$ is the receiver gain. The algebraic manipulation of the above relations yields the following transfer dynamics:

$\frac{V_{R}}{\dot{\sigma}}=\frac{1 / \mathrm{K}}{1+\tau_{R} s}$

where: $\tau_{R}=1 /\left(\mathrm{KK}_{\mathrm{R}}\right)$ is the receiver time constant. Thus, the radar output voltage is proportional to the rate of rotation of the line of sight but with a time lag inversely proportional to the seeker gain $\left(K_{R}\right)$. Large values of this gain yield small lag and consequently the antenna is closely tracking the target. Assuming that the antenna axis is accurately pointing to the target i.e. $\varepsilon=0$, consequently $\dot{\sigma}=K_{R}$ where $1 / \mathrm{K}=0.45 \mathrm{~N}_{\text {gain }}$ and $\mathrm{N}_{\text {gain }}$ is dependent on the variation of the closing velocity $\left(\mathrm{V}_{\mathrm{c}}\right)$ as follows [14]:

$$
\mathrm{N}_{\text {yain }}= \begin{cases}0.33+\frac{0.17}{770} \mathrm{~V}_{\mathrm{c}} & \mathrm{V}_{\mathrm{c}}<750[\mathrm{~m} / \mathrm{sec}] \\ \frac{0.5}{770} \mathrm{~V}_{\mathrm{c}} & \mathrm{V}_{\mathrm{c}} \geq 750[\mathrm{~m} / \mathrm{sec}]\end{cases}
$$

The guidance command is generated by adding the seeker rate gyro output to the seeker tachometer output. This guidance command is a voltage describing the required normal acceleration for the missile to follow the target and it is limited to the maximum missile maneuver. The generation sequence of guidance commands is shown in Fig. (6), where the pitch (yaw) command is generated according to the following equation:

$\eta_{p c}=A\left(\dot{\sigma}_{p} / k+\dot{\lambda}_{p} F_{2}\right)$

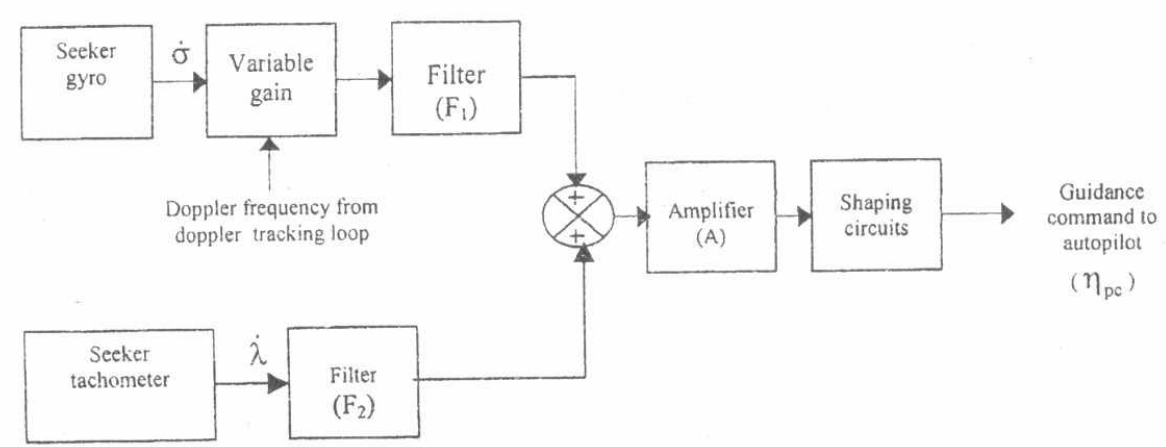

Fig. (6): Generation sequence of guidance commands 


\subsection{Aerodynamic Transfer Functions}

Investigating the performance of the missile guidance and control system necessitates the determination of the missile airframe dynamics in the pitch and yaw planes. The two planes are similar, and consequently one plane is considered for the subsequent discussions. In addition, the perturbation approach is used to linearize the guidance equations around certain trim conditions.

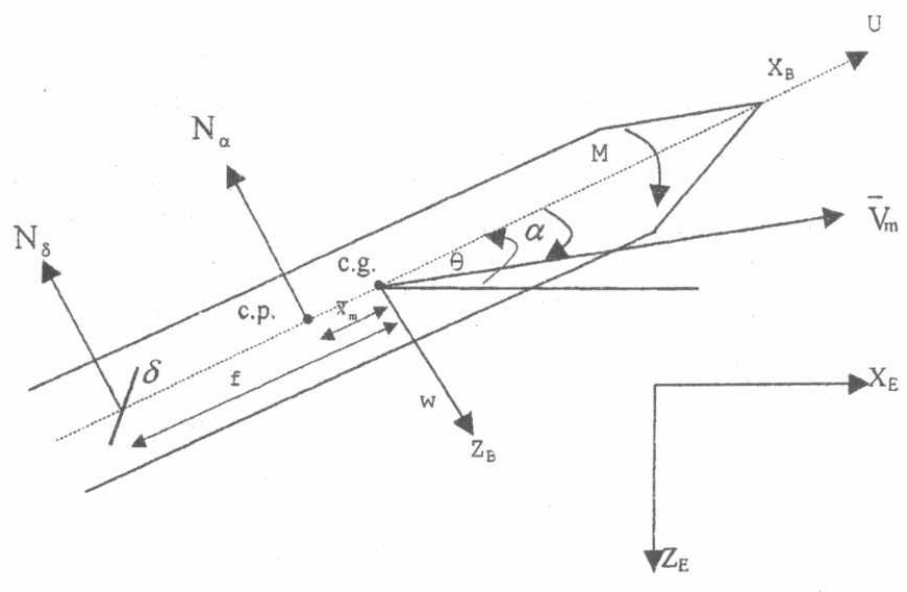

Fig. (7): Missile in pitch plane

Fig. (7) shows a free body diagram of the missile in the pitch plane, where the angle of attack is defined by $\alpha=\tan ^{-1} \frac{\mathrm{w}}{\mathrm{U}} \quad \alpha=\frac{\mathrm{w}}{\mathrm{U}}$. Using the linearized equations with zero initial conditions, it is possible to obtain transfer functions for the missile airframe as follows:

$\mathrm{m} \eta_{\mathrm{z}}(\mathrm{s})+\mathrm{N}_{\alpha} \alpha(\mathrm{s}) \quad=-\mathrm{N}_{8} \delta(\mathrm{s})$

$\eta_{\mathrm{z}}(\mathrm{s})-\mathrm{U}_{\mathrm{o}} \mathrm{s} \alpha(\mathrm{s})+\mathrm{U}_{0} \dot{\theta}(\mathrm{s})=0$

$\left(\mathrm{N}_{\alpha} \overline{\mathrm{x}}_{\mathrm{m}}-\mathrm{sM}_{\dot{\alpha}}\right) \alpha(\mathrm{s})+\mathrm{Is} \dot{\theta} \dot{\mathrm{s}}(\mathrm{s})=-\mathrm{N}_{\delta} \mathrm{f} \delta(\mathrm{s})$

These equations can be arranged in the following matrix form:

$\left[\begin{array}{ccc}\mathrm{m} & \mathrm{N}_{\alpha} & 0 \\ 1 & -\mathrm{U}_{\mathrm{o}} \mathrm{s} & \mathrm{U}_{\mathrm{o}} \\ 0 & \mathrm{~N}_{\alpha} \overline{\mathrm{x}}_{\mathrm{m}}-\mathrm{s} \mathrm{M}_{\dot{\alpha}} & \mathrm{Is}_{\mathrm{s}}\end{array}\right]\left[\begin{array}{c}\eta_{\mathrm{z}}(\mathrm{s}) \\ \alpha(\mathrm{s}) \\ \dot{\theta}\end{array}\right]=\left[\begin{array}{c}-\mathrm{N}_{\delta} \delta(\mathrm{s}) \\ 0 \\ -\mathrm{N}_{\delta} \mathrm{f} \delta(\mathrm{s})\end{array}\right]$

Applying Cramer's rule [7] to solve these equations for $\dot{\theta}(\mathrm{s}), \alpha(\mathrm{s})$ and $\eta_{2}(\mathrm{~s})$, the following missile airframe transfer functions in pitch plane are obtained as follows [10]:

$$
\begin{aligned}
& \frac{\dot{\theta}(s)}{\delta(s)}=-\frac{N_{\delta}}{I} \frac{\left(f-\frac{M_{\dot{\alpha}}}{m U_{0}}\right) s+\frac{f-\bar{x}_{m}}{m U_{0}} N_{\alpha}}{s^{2}+\left(\frac{N_{\alpha}}{m U_{0}}+\frac{M_{\dot{\alpha}}}{I}\right) s+\frac{\bar{x}_{m} N_{\alpha}}{I}} \\
& \frac{\alpha(s)}{\delta(s)}=-\frac{N_{\delta}}{m}-\frac{U_{0} s+\frac{m f}{I}}{s^{2}+\left(\frac{N_{\alpha}}{m U_{0}}+\frac{M_{\dot{\alpha}}}{I}\right) s+\frac{\bar{x}_{m} N_{\alpha}}{I}} \\
& \frac{\eta(s)}{\delta(s)}=-\frac{N_{\delta}}{m} \cdot \frac{s^{2}+\frac{M_{\dot{\alpha}}}{I} s+\frac{\bar{x}_{m}-f}{I} N_{\alpha}}{s^{2}+\left(\frac{N_{\alpha}}{m U_{0}}+\frac{M_{\dot{\alpha}}}{I}\right) s+\frac{\bar{x}_{m} N_{\alpha}}{I}}
\end{aligned}
$$

The aerodynamic characteristics of the missile vary during its flight due to the change 
of missile speed, incidence angles, missile mass, moments of inertia, altitude, etc. Thus for each instant there is different transfer function describing the aerodyramic characteristics of the missile. That is for evaluating those transfer dynamics, a certain engagement scenario is considered and different instants related to the Mach number are chosen [8]. For example, considering a scenario with target flying at initial position $\left[X_{T}, Y_{T}, Z_{T}\right]=\left[\begin{array}{lll}6 & 1 & 2\end{array}\right][\mathrm{Km}]$ and speed $\left[V_{X_{T}} V_{Y_{T}} V_{Z_{T}}\right]=\left[\begin{array}{llll}-100 & -200 & 0\end{array}\right][\mathrm{m} / \mathrm{sec}]$, and according to the obtained flight parameters of this scenario and the transfer functions derived before, the numerical results at same flight condition $(t=6 \mathrm{sec})$ are:

\begin{abstract}
$\frac{\dot{\theta}_{\mathrm{F}}(\mathrm{s})}{\delta_{\mathrm{F}}(\mathrm{s})}=\frac{245 \mathrm{~s}+1.01 \times 10^{4}}{\mathrm{~s}^{2}+1.315 \mathrm{~s}+186}$
$\frac{\eta_{F}(s)}{\delta_{F}(s)}=\frac{5264 s^{2}+294 s-6.791 \times 10^{6}}{s^{2}+1.315 s+186}$

The step response for the aerodynamic characteristics of the missile airframe at $t=6 \mathrm{sec}$, and $M=1.9635$ due to wing deflection is shown in Fig. (8). This figure shows the small damping ratio in addition to the small static stability of the missile. Thus, it is necessary to include an autopilot for improving the dynamic stability of the system, which designed and analyzed in the following section.
\end{abstract}

$$
\begin{aligned}
& \frac{\dot{\theta}_{W}(s)}{\delta_{W}(s)}=\frac{36.42 \mathrm{~s}+141.1}{\mathrm{~s}^{2}+1.315 \mathrm{~s}+186} \\
& \frac{\eta_{W}(\mathrm{~s})}{\delta_{W}(\mathrm{~s})}=\frac{671.3 \mathrm{~s}^{2}-37.49 \mathrm{~s}-9.419 \times 10^{4}}{\mathrm{~s}^{2}+1.315 \mathrm{~s}+186}
\end{aligned}
$$

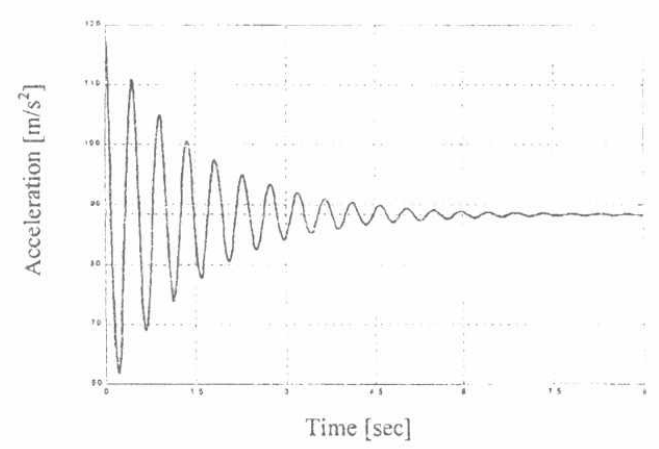

Fig. (8): Acceleration step response due to wing deflection at $6 \mathrm{sec}$

\section{4- Control System Design and Analysis}

During the sustain phase, both fin and wing channels are active yieiding the coupled system operation as shown in Fig. (9). Therefore, this section is devoted to investigate its performance in addition to any required designs for enhancement. The fins are used to generate and contro! the attitude-rate because of their physical location on the missile. In addition, the deflection of fins causes the missile to accelerate as well. This acceleration must therefore be combined with the acceleration caused by the deflection of wings. Similarly, the wings are used to provide the dominant missile lateral acceleration. The distance from the wings hinge line to the center of mass is a moment arm and indicates that wing deflections will also cause the missile to rotate around its center of mass generating an angular rate. Thus, an accurate description of the missile rotational motion requires that the angular rate due to wings deflection be summed with that generated by the deflection of fins. The system response for a unit step input is shown in Fig. (10), where the system is relatively slow stable with rise time of about $1.75 \mathrm{sec}$. This nature of response is not required in high maneuver missiles $[10,12]$. 


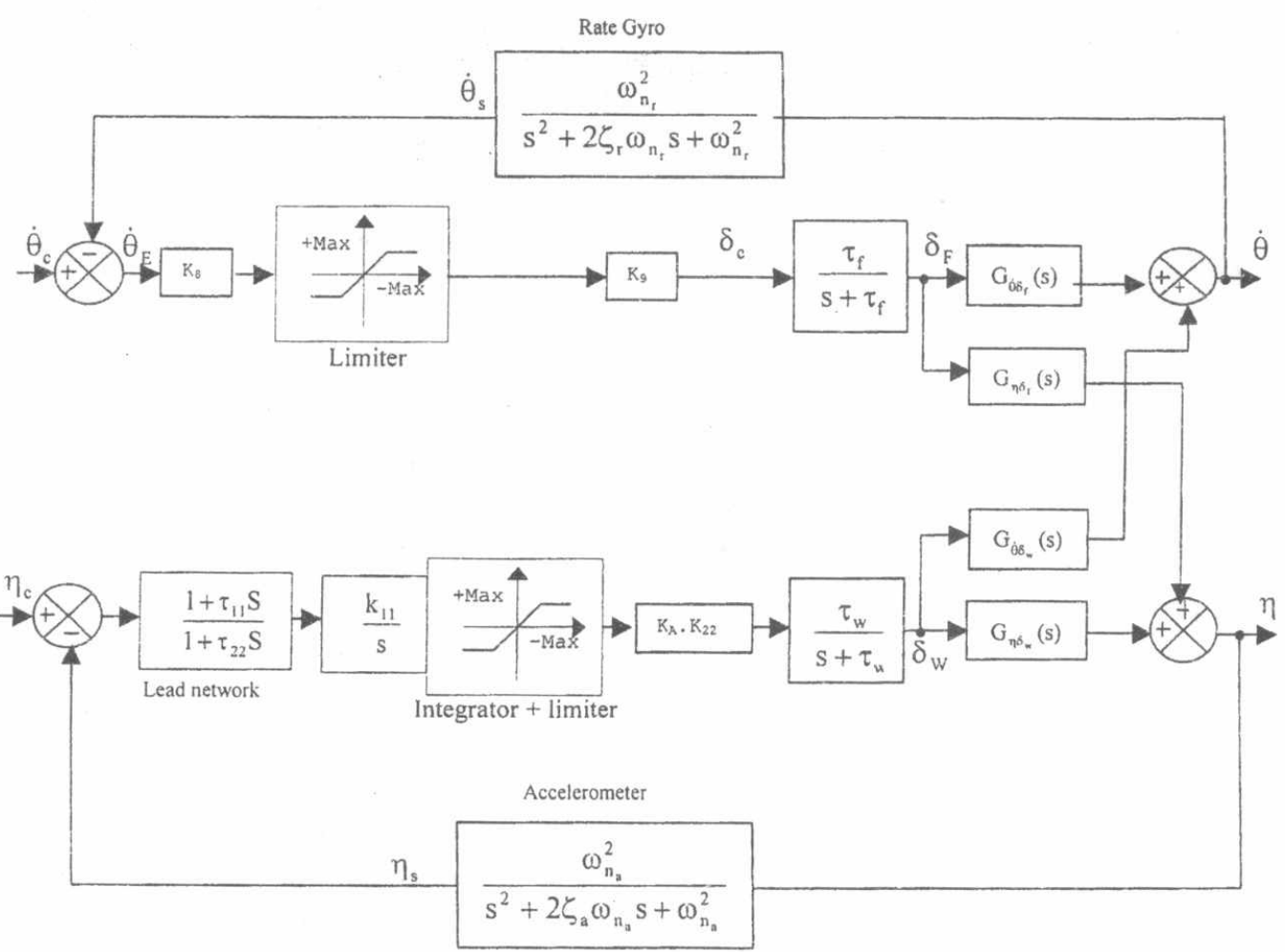

Fig. (9): The coupled control system

Therefore, a Proportional-Integral-Derivative (PID) compensator circuit is used to enhance the system performance, and it is tuned with a trade off technique yielding the following form:

$\mathrm{TF}=\mathrm{K}_{\mathrm{P}}+\frac{\mathrm{K}_{\mathrm{I}}}{\mathrm{s}}+\mathrm{K}_{\mathrm{D}} \mathrm{s}$

where : $K_{p}=3.329$,

$K_{1}=0.0591$, and $K_{D}=0.54$.

Then, the system

response to a step

command is shown in

fig. (11), where the rise

time is reduced by $87 \%$,

yielding a relatively fast response.

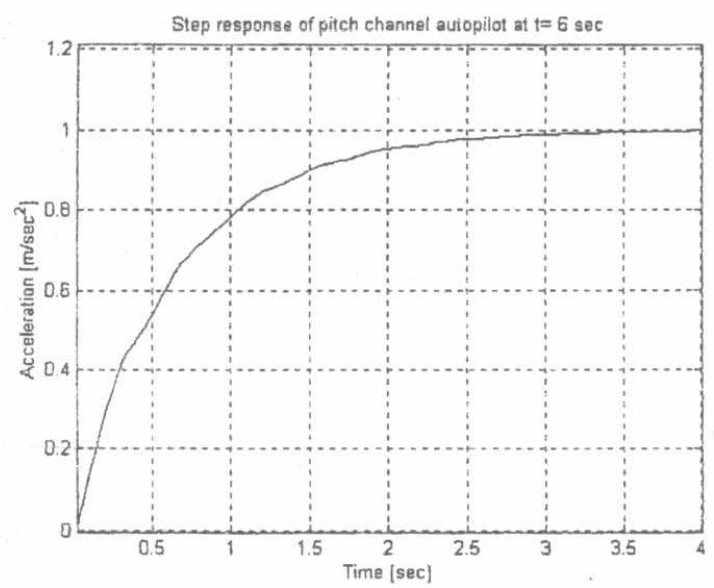

Fig. (10):"Step response of pitch channel autopilot at $\mathrm{t}=6 \mathrm{sec}$ with lead network 
Proceedings of the $10^{\text {th }}$ ASAT Conference, 13-15 May 2003

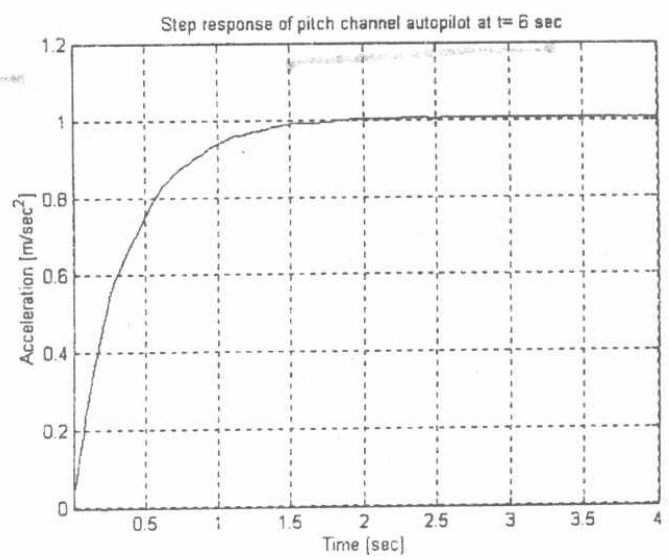

Fig. (11): Step response of missile autopilot at $\mathrm{t}=6 \mathrm{sec}$ with PID
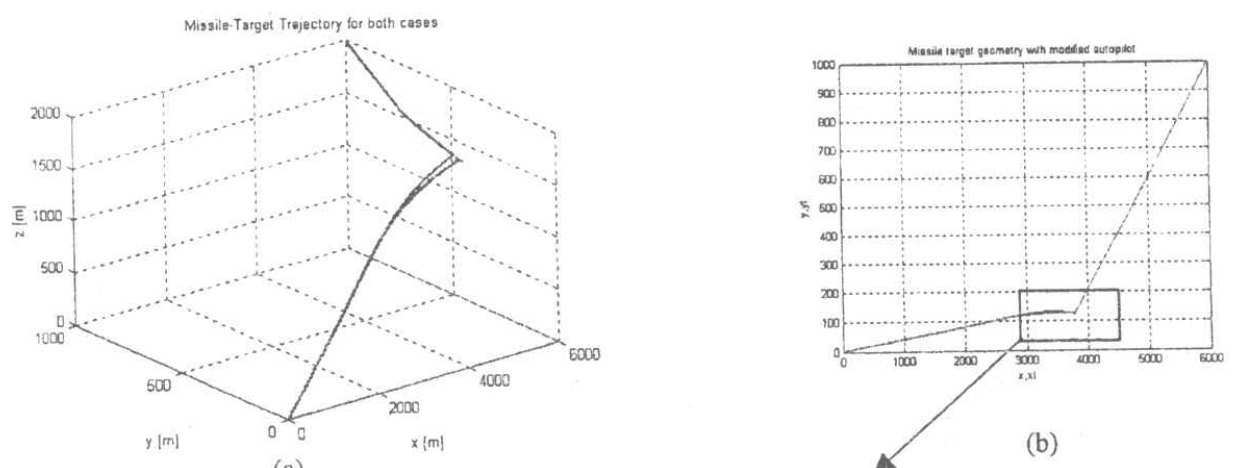

(a)

Missile target geometry with modified autopilot

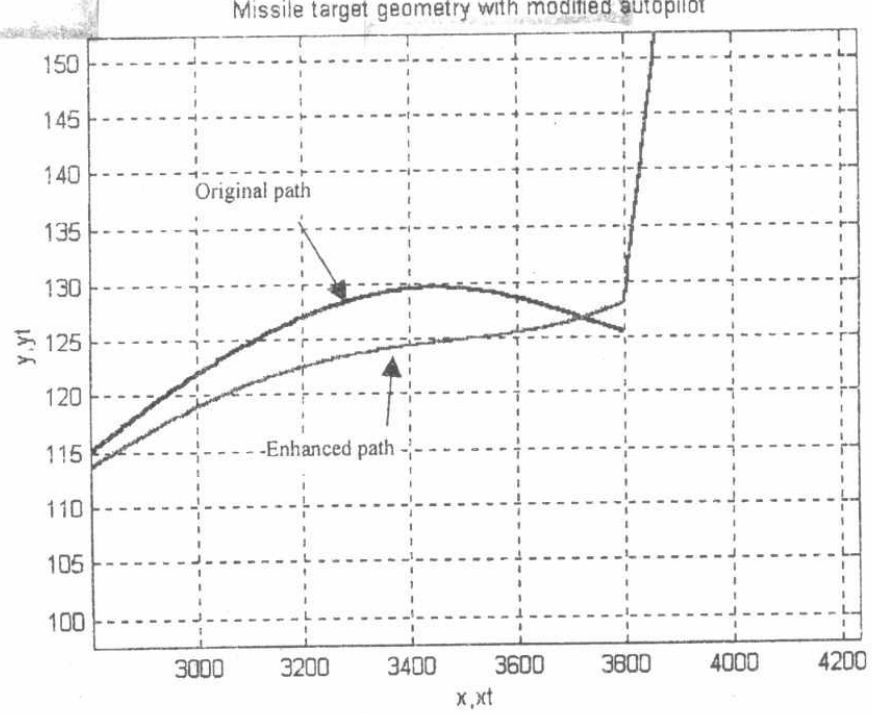

(c)

Fig. (12): Missile-target engagêment scenarios with lead and PID networks 
To insure the robustness of the proposed PID controller; the 6DOF simulation model is

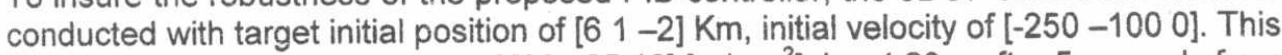
target experienced a maneuver of $[30-2510]\left[\mathrm{m} / \mathrm{sec}^{2}\right]$, i.e. $4.23 \mathrm{~g}$ after 5 seconds from the instance of missile launch, and lasted for 2 seconds. The missile-target flight path with the two controllers is shown in Fig. $(12-a)$ where the miss distance is $47.8[\mathrm{~m}]$ and the time of flight is 8.27 seconds in case of the original lead network. While, using the PID the miss distance is about $20[\mathrm{~m}]$, and the flight time is 8.26 seconds. The new system is much faster than the original one, and with less miss distance of about $58 \%$. The two engagement scenarios are plotted together with zooming to clarify the difference between them as shown in Fig. (12-b, 12-c).

\section{5- Conclusions}

This paper presented a brief description for the 6DOF model of the underlying guidance system and its utilization for subsequent design and analysis. Numerical implementation with different engagement scenarios and the known guidance method, toward which a linearized model for the missile airframe dynamics is developed. The approach utilized in analyzing the missile control system was initially to investigate the two channels of control independently (decoupled) including the forward (wings) acceleration control system and the rear (fins) attitude/attitude-rate control systems. These two control systems are coupled through the airfame dynamics. The step response of the coupled system showed a stable system but with a very large settling time, which is compensated with a PID controller. The robustness of this controller, is justified via the 6DOF simulation model where the miss distance was decreased with about $58 \%$.

\section{References}

[1] Asher R. B. and J. P. Matuszewski, Optimal quidance with maneuvering targets, Journal Spacecraft, 11(3), 204-206, 1973

[2] Bryson A. E., Jr. and Y. Ho, Applied Optimal Control. Hemisphere, Washington D.C., U.S.A, 1975.

[3] Bugajski D., D. Enns, R. Henrick, C. Russ, Flight Control Laws for the HARV, Phase 2 Final Report, March 1992.

[4] Carnahan B., H. A. Luther, and J. O. Wilkes, Applied Numerical Methods, John Wiley \& Sons Inc., New York, U.S.A., 1969

[5] Cottrell R. G., Optimal intercept guidance for short-range tactical missile, AIAA Journal, 9, 14141415,1971

[6] El-Sheikh G.A., Theory Of Guidance And Systems, Lecture Notes, SN1898, Military Technical College, 2000.

[7] Etkin B. and L. Reid, Dynamics of Flight Stability and Control, Wiley, 1996.

[8] Frazzoli E., M.A. Dahleh, E. Feron, Robust Hybrid Control for Autonomous Systems Motion Planning, Technical report LIDS-P-2468, Laboratory for Information and Decision Systems, Massachusetts Institute of Technology, Cambridge, MA, 1999.

[9] Garnell P., and East D. J., Guided Weapon Control Systems, Pergamon Press, Oxford, England, 1977.

[10] John $\mathrm{H}$. Bakelock, Automatic control of aircraft and missiles, A Wiley-Interscience Publication, $2^{\text {nd }}$ Edition, 1991.

[11] Kreindler, E., Optimality of proportional navigation, AIAA Journal, 11, 878-880, 1973

[12] Nazaroff G. J., An optimal terminal guidance law, IEEE Transaction on Automatic Control, AC$21(1), 407-408,1976$.

[13] Nesline F. W., and N. C. Nabbefeld, Design of Digital Autopilots for Homing Missiles, AGARD Flight Mechanics Panel Symposium, pp. 29-1-29-14, 1979.

[14] Nesline F. W., B. H. Wells, and P. Zarchan, A Combined Optimal/Classical Approach to Robust Missile Autopilot Design, AIAA Guidance and Control Conference, AIAA, New York, pp. 265-280, 1979.

[15] Richard Y. Chiang, Michael G. Safonov, MATLAB Robust Control Toolbox User's Guide, The Math Works, inc. 1998. 